\title{
The relativity of age or geriatric medicine at the crossroads
}

\author{
Gorazd Voga · Dragan Kovačić
}

Received: 24 October 2016 / Accepted: 31 October 2016 / Published online: 21 November 2016 (C) Springer-Verlag Wien 2016

The age of the Universe is estimated at 13.8 bn years and our current belief is that the Earth is $4.5-4.6 \mathrm{bn}$ years old [1, 2]. Modern humans evolved about 200,000 years ago and therefore our history represents only a minor part of the old Earth and Universe. According to the World Health Organization statistics (WHO 2016), the average life expectancy at birth in the world population for 2015 was 71.4 years, the lowest being in Sierra Leone (50.1 years) and the highest in Japan (83.7 years) [3]. It is worth emphasizing that the average life expectancy for those born in 1965 was 53.3 years while 100 years ago, it was only approximately 35 years. Furthermore, in the millennia from B.C. up to the nineteenth century, the life expectancy remained very stable with only minor fluctuations. Therefore, in the last 150 years we are facing rapid ageing of the population in both developed countries and in those undergoing transition. The life expectancy in Europe, e.g. increased in the past century by 35-50 years. Ageing of the population is not only the success of the modern way of life, well-being and progress of health sciences but at the same time causes many medical, psychological, sociological and financial problems $[4,5]$. It is associated with typical geriatric syndromes (e.g. sleep problems, delirium, dementia, osteoporosis, heart failure, falls and weight loss) that reduce the health-related quality of life, activities of daily living and therefore increase their dependence and need for various forms of sup-

Assoc. Prof. G. Voga, MD, PhD (ه)

Dpt. of Intensive Internal Medicine, General Hospital Celje, Oblakova 5, 3000 Celje, Slovenia

gorazd.voga@guest.arnes.si

D. Kovačić, MD, PhD

Dpt. of Cardiology, General Hospital Celje,

Oblakova 5, 3000 Celje, Slovenia

dragan.kovacic@guest.arnes.si port [6-8]. The elderly frequently suffer from agerelated vulnerability due to reduced compensatory and defence mechanisms that leads to frailty with associated polymorbidity and polypharmacy, undernutrition, poor social status, loneliness and repetitive hospital admissions [9-11]. Once hospitalized, the main goal unfortunately remains rapid management of acute medical conditions. Even though older patients have a prolonged hospital stay and are at higher risk for hospital-acquired infections and death, discharge from hospital is often premature and with little concerns about the transition to domestic or nursing long-term facilities.

There is abundant scientific and non-scientific evidence that human societies and modern medicine are simply not prepared and adapted to the aging of the population. In clinical practice, the age itself is a major hindrance to standards of care. If admitted to the emergency facilities or intensive care unit (ICU), old and very old patients often do not get adequate diagnostic work-up and treatment as compared with younger patients. In patients with acute myocardial infarction, the elderly have longer prehospital delay before treatment and they are less likely to receive the recommended medical and interventional care $[12,13]$. The proportion of older patients admitted to the ICU is increasing. Nevertheless, only $40 \%$ of patients older than 80 years with fulfilled criteria for ICU admission were referred to the ICU and only half of them were finally admitted [14]. Differences in treatment activities (e.g. mechanical ventilation, renal replacement therapy and vasopressors) between younger and older patients were reported but management intensity of older patient treatment improved in last decade and is probably related with a better outcome [15]. Crucial to that is the diagnostic process and initial decision-taking with therapeutic implications and evidence is emerging that the elderly 
are not withheld key management steps. Prospective, observational study of triage decisions, performed in 11 ICUs in 7 European countries showed that elderly patients have more ICU rejections and higher mortality than younger ones. On the other hand, differences between mortality of accepted versus rejected patients were greatest for older patients indicating that the benefit of ICU admission appears to be greater for the elderly [16, 17]. Despite the fact that age is an important and independent predictor of mortality, it cannot be used as the main admission criterion, as the severity of an acute medical condition is much more strongly associated with mortality [18].

Therefore, the emergency ward and ICU management of the acute medical conditions should not depend on age but needs to be tailored to the patient in line of standards of care.

Problems that are even more important emerge after patient is stabilized and the discharge process is in sight. Most elderly patients require complex and prolonged rehabilitation, which, in spite of medical indications, is frequently not provided because of economically oriented insurance funds and inadequate knowledge of the medical professionals, who should be able to provide a holistic and individualized management of elderly patients [19]. The advantages of such multidisciplinary management are shorter hospital stay, appropriate rehabilitation, organized transition from hospital to home, reduced readmissions and reduced costs. A meta-analysis of 17 randomized trials evaluating geriatric rehabilitation units found that inpatient multidisciplinary programs were associated with improvement in all outcomes at discharge, including better functional status, decreased nursing home admission and lower mortality rate [20]. Another meta-analysis of 22 randomized trials found that hospitalized patients receiving comprehensive geriatric evaluation in specialized geriatric units were more likely to be alive and in their homes at 6-month and 12-month follow-up [21].

After successful treatment of acute illness, elderly people should therefore receive complex and prolonged physical, social and psychological rehabilitation. Nevertheless, we must be able to recognize the point of futile treatment and provide proper palliative care.

Preventive programs, such as promotion of healthy lifestyles are very important and successful but they must also include reduction of the negative social attitude and assure the psychosocial integration of the elderly. Prevention and treatment of delirium, which is the most frequent complication following hospitalization, is possible and of utmost importance [22]. As cardiovascular and malignant diseases are the most frequent causes of death in older patients, new, more effective and less traumatizing treatments are beneficial in geriatric patients. A typical example is the transcatheter aortic valve implantation (TAVI) in el-

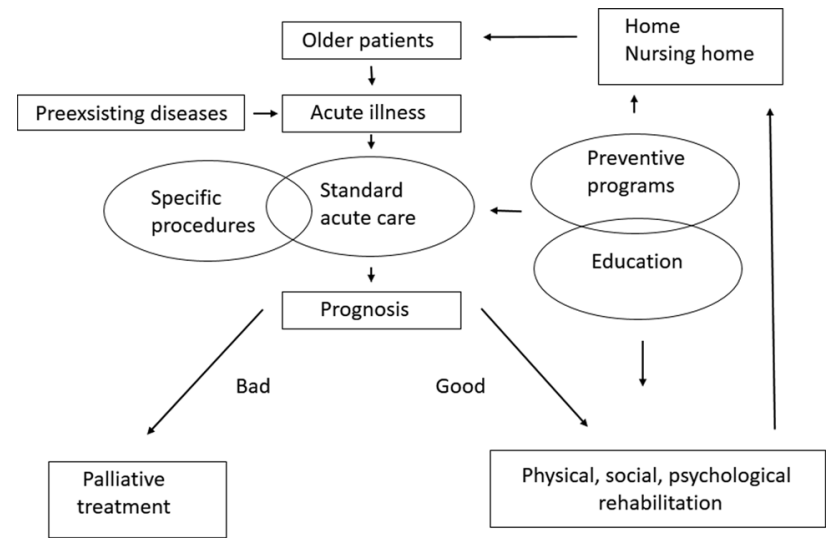

Fig. 1 In the case of acute illness, geriatric patients should receive standard acute treatment (or specially adapted procedures) mainly according to the severity of the acute conditions. Patients with good prognosis should enter prolonged rehabilitation programs and proper socialization. Patients with futile treatment and poor prognosis should receive proper palliative care. Preventive programs and education are essential parts of the management

derly patients with severe aortic stenosis who are at excess risk of complications with open-heart surgery. Older patients receiving TAVI have favorable in-hospital recovery and similar mortality compared to standard management; therefore, TAVI is a more beneficial and tolerable procedure in very old patients with similar complication rates than in younger patients [23].

General preventive programs promoting healthy lifestyle have been developed but must be implemented in the majority of older people. Less traumatic procedures, which are better tolerated, are preferred in the management of specific medical conditions in geriatric patients.

Geriatric patients require management by a multidisciplinary approach with active participation of specially trained geriatric specialists [24]. Up to now, healthcare has not been prepared to meet the burden and challenges of increased numbers and complex management in the elderly. The distribution of geriatric medicine is unequal, geriatric medicine is not a mandatory part of undergraduate medical school programs and the speciality of geriatric medicine is still not recognized in many countries [25]. The pharmacological therapy, in this context, is a specific challenge due to several issues. The evidence for many guideline-conform therapies is less solid for the elderly than for younger patients [26]. In clinical practice, guideline-based (poly) pharmacy due to several chronic conditions gives a perfect foundation for drug-drug interactions [27]. Even very simple systems can be helpful and generally, it holds true that physicians with training in geriatrics are less likely to prescribe potentially inappropriate medications [28, 29]. These issues need to be given first line priority by the decision makers to allow adequate development 
of geriatric medicine at all levels of the healthcare system.

Medical science should promote adequate education of all professionals, who are involved in the treatment of geriatric patients; societies should provide equal access to healthcare in developed countries and countries in transition.

Conflict of interest G. Voga and D. Kovacic declare that they have no competing interests.

\section{References}

1. Redd NT. How old is the universe? 2013. http://www.space. com/24054-how-old-is-the- universe.html. Last access: 27.09.2016.

2. Braterman PS. How science figured out the age of the earth 2013. http://www.scientificamerican.com/article/howscience-figured-out-the-age- of-the-earth/. Last access: 27.09.2016.

3. WHO. Life expectancy 2016. http://www.who.int/gho/ mortality_burden_disease/life_tables/situation_trends_ text/en/. Last access: 27.09.2016.

4. Ribera Casado JM. The history of geriatric medicine. The present: problems and opportunities. Eur Geriatr Med. 2012;3:228-32.

5. Christensen K, Doblhammer G, Vaupel JW. Ageing population: the challenges ahead. Lancet. 2009;374:1196-208.

6. Morley JE. Frailty and sarcopenia in elderly. Wien Klin Wochenschr. 2016; doi:10.1007/s00508-016-1087-5.

7. Brilej D, Krušič D, Komadina R, Currie C. Audit of geriatric hip fracture care-a Slovenian trauma center analysis. Wien Klin Wochenschr. 2016;WKWO-D-16-336R1.

8. Omersa D, Lainscak M, Erzen I, Farkas J. Mortality and readmissions in heart failure: an analysis of 36,824 elderly patients from Slovenian national hospitalization database. Wien Klin Wochenschr. 2016; doi:10.1007/s00508-0161098-2.

9. Fries JF. Measuring and monitoring success in compressing morbidity. Ann Intern Med. 2003;139:455-9.

10. Fried L, Walston J. Frailty and failure to thrive. In: Hazzard W, Blass JP, Halter JB, Ouslander JG, Tinetti ME, editors. Principles of geriatric medicine and gerontology, 5th edn. NewYork: McGrawHill; 2003. pp. 1487-502.

11. Bauer JM, Sieber CC. Sarcopenia and frailty: a clinician's controversial point of view. Exp Gerontol. 2008;43:674-8.

12. Nguyen HL, Saczynski JS, Gore JM, Goldberg RJ. Age and sex differences in duration of prehospital delay in patients with acute myocardial infarction: a systematic review. Circ Cardiovasc Qual Outcomes. 2010;3:82-92.

13. Schoenenberger AW, Radovanovic D, Stauffer JC, et al. Agerelated differences in the use of guideline-recommended medical and interventional therapies for acute coronary syndromes: a cohortstudy. JAm Geriatr Soc. 2008;56:510-6.
14. Garrouste-Orgeas M, Boumendil A, Pateron D, et al. Selection of intensive care unit admission criteria for patients aged 80 years and over and compliance of emergency and intensive care unit physicians with the selected criteria: an observational, multicenter, prospective study. Crit Care Med. 2009;37:2919-28.

15. Lerolle N, Trinquart L, Bornstain C, etal. Increased intensity of reatment and decreased mortality in elderly patients in an intensive care unit over a decade. Crit Care Med. 2010;38:59-64.

16. Voga G, Gabršček-Parežnik L. Initial hemodynamic assessment and treatment of elderly patients in medical ICU. Wien Klin Wochenschr. 2016;WKWO-D-16-00438.

17. Sprung CL, Artigas A, Kesecioglu J, et al. The Eldicus prospective, observational study of triage decision making in European intensive care units. Part II: intensive care benefit for the elderly. Crit Care Med. 2012;40:132-8.

18. Brunner-Ziegler S, Heinze G, Ryffel M, Kompatscher M, Slany J, Valentin A. "Oldest old" patients in intensive care: prognosis and therapeutic activity. Wien Klin Wochenschr. 2007;119:14-9.

19. Tulloch AJ. Effectiveness of preventive care programmes in the elderly (editorial). Age Ageing. 2005;34:203-4.

20. BachmannS, Finger C, Huss A, et al. Inpatientrehabilitation specifically designed for geriatric patients: systematic review and meta-analysis of randomised controlled trials. BMJ. 2010;340:c1718.

21. Ellis G, Whitehead MA, O'Neill D, et al. Comprehensive geriatric assessment for older adults admitted to hospital. Cochrane Database Syst Rev. 2011;2011:CD006211.

22. Young J, Cheater F, Collinson M, et al. Prevention of delirium (POD) for older people in hospital: study protocol for a randomised controlled feasibility trial. Trials. 2015;16:340-51.

23. Alsara O,AlsarahA, Laird-FickH.Advanced age and the clinical outcomes of transcatheter aortic valve implantation. J Geriatr Cardiol. 2014;11:163-70.

24. Nguyen KL, Alrezk R, Mansourian PG, Naeim A, Rettig MG, Lee CC. The crossroads of geriatric cardiology and cardiooncology. Curr Geriatr Rep. 2015;4:327-37.

25. Michel JP, Huber P, Cruz-Jentoft AJ. Europe-wide survey of teaching in geriatric medicine. J Am Geriatr Soc. 2008;56:1536-42.

26. Vitale C, Rosano G, Fini M. Are elderly and women underrepresented un cardiovascular clinical trials? Implications for treatment. Wien Klin Wochenschr. 2016; doi:10.1007/ s00508-016-1082-x.

27. Roblek T, Trobec K, Mrhar A, Lainscak M. Potential drugdrug interactions in hospitalized patients with chronic heart failure and chronic obstructive pulmonary disease. Arch Med Sci. 2014;10:920-32.

28. Roblek T, Vaupotic T, Mrhar A, Lainscak M. Drug-drug interaction software in clinical practice: a systematic review. Eur JClin Pharmacol. 2015;71:131-42.

29. Stock S, Redaelli M, Simic D, Siegel M, Henschel F. Risk factors for the prescription of potentially inappropriate medication (PIM) in the elderly. Wien Klin Wochenschr. 2014;126:603-12. 